\title{
CAPTURE DES LARVES DE CULICIDÉS PAR LES PLANTES
}

\section{DU GENRE UTRICULARIA}

\author{
Par E. BRUMPT
}

Le nombre de moustiques constaté dans une même région d'une année à l'autre dans des conditions climatiques comparables est à peu près constant. Pourtant, par suite de la fécondité des femelles, leur nombre devrait être multiplié théoriquement par cinq cents ou mille chaque année. Cette différence tient à l'action exercée, dans le milieu ambiant, par des facteurs physiques défavorables et surtout par les ennemis naturels des adultes et des larves.

Je ne m'occuperai dans cette courte communication que du rôle de certains végétaux, généralement aquatiques, appartenant au genre Utricularia.

Diverses plantes ont été préconisées pour lutter contre les larves de moustiques. Les unes comme les lentilles d'eau (Lemna, sp. sp.), les Pistia, les Limnobium, les Eichornia, les Piaropus (1), les Azolla, les Salvinia et un grand nombre d'autres plantes peuvent, quand elles forment une couverture complète à la surface de l'eau, empêcher le développement des anophèles. Certaines autres, comme diverses Characées, dont le rôle est d'ailleurs contesté par plusieurs auteurs, vivent dans des eaux qui, par leur composition chimique ou par leur flore, ne conviennent peut-être pas aux larves d'anophèles.

Dans le cas des utriculaires (2) dont certaines espèces flottent entre deux eaux dans les mares à eau tranquille, claire, ou rendue trouble par du limon en suspension, le mode d'action est tout à fait différent. Ces plantes peuvent en effet se multiplier dans les eaux où le développement des larves de moustiques s'effectue normalement, elles peuvent même détruire un assez grand nombre de ces dernières en les emprisonnant dans leurs vésicules.

L'Utricularia vulgaris, que j'ai étudiée aux environs de Paris, est dépourvue de racines; elles apparait au printemps, longtemps avant l'éclosion des premières larves d'Anopheles maculipennis et se rencontre à l'arrière-saison en novembre quand la température

(1) En Louisiane certains cours d'eau sont entièrement couverts par les « Water hyacinth 》) (=Piaropus crassipes).

(2) Ce genre de plantes fait l'objet d'une êtude biologique et systématique, publiée dans ce même numéro par le Dr M. Langeron.

Annales de Parasitologie, $\mathrm{T}$. III, No 4 . - Octobre 1925, p. 403-411. 
n'est pas trop rigoureuse. Pendant la saison froide, cette plante est réduite à des bourgeons ou hibernacles, qui tombent au fond des mares et qui, dès l'approche du printemps, remontent à la surface, se développent rapidement et présentent alors de nombreux utricules grands et petits, capables d'absorber des proies proportionnées à leurs dimensions.

Dans les lignes qui suivent, je vais signaler, après avoir donné un aperçu historique, le rôle intéressant que les utriculaires peuvent jouer dans la lutte contre les moustiques et dans la prophylaxie du paludisme.

Historique. - La propriété de capturer des proies vivantes présentée par les vésicules des utriculaires semble avoir été signalée pour la première fois par le botaniste français Crouan (1) qui, en 1858 , constata la présence de petits crustacés dans celles de l'Utricularia vulgaris.

Dix ans plus tard, Holland (1868) signale, en Angleterre, la présence d'insectes aquatiques dans les vésicules d'une jtriculaire et émet l'opinion que cette plante peut en tirer quelques substances alimentaires.

Dans Utricularia vulgaris, Cohn (1875) observe divers crustacés et vers d'eau douce.

Dans les vessies d'Utricularia clandestina de New-Jersey, Mme Treat (1875) signale des crustacés et très souvent des larves de culicides.

Egalement en 1875 , Ch. Darwin, dans son remarquable ouvrage sur les plantes insectivores, dit avoir rencontré des Cypris et des copépodes, parfois au nombre de dix, chez Utricularia vulgaris ; divers crustacés entomostracés et des larves d'insectes chez Utricularia neglecta; enfin parfois jusqu'à vingt crustacés plus ou moins reconnaissables dans une seule vésicule d'Utricularia minor. Dans ce même ouvrage, Ch. Darwin résume les observations qu'il a faites en étudiant des échantillons exotiques conservés dans les herbiers du jardin botanique de Kew. Dans les utricules développés sur les rhizomes de l'Utricularia montana, espèce terrestre, il trouve des débris de petits animaux.

Dans les vésicules d'Utricularia nelumbifolia (2), il trouve des

(1) Cité par Darwin, d'après Delpino.

(2) Cette curieuse espèce, découverte dans les montagnes des Orgues (Etat de Rio de Janeiro, Brésil) par Gardner (1836-1841), vit dans l'eau collectée par les broméliacés du genre Tillandsia croissant sur les roches arides à une altitude d'environ 1.500 mètres. Comme l'eau accumulée entre les gaines de leurs feuilles par diverses Tillandsia (Broméliacées) renferme des larves d'un certain nombre d'espèces de moustiques, pathogènes ou non, il serait intéressant de savoir si ces dernières sont détruites par les vésicules de cette utriculaire. 
débris de divers arthropodes, ainsi que dans celles des espèces suivantes : $\boldsymbol{U}$. amethystina de la Guyane, $\boldsymbol{U}$. griffithsi de Malaisie et de Bornéo, $U$. cærulea, $U$. orbiculata, $U$. multicaulis des Indes anglaises et de plantes voisines des utriculaires comme les Polypompholyx multifida de l'Australie occidentale et les Genlisea ornata du Brésil et $G$. africana de l'Afrique méridionale.

En 1882, Schimper a observé chez Utricularia resupinata, $U$. subulata et surtout chez $U$. cornuta, espèces terrestres, des rotifères, des vers, des crustacés, des grains de sable, des carapaces de diatomées et des infusoires.

En 1890, Gœbel a signalé dans les vésicules d'U. orbiculata, espèce épiphyte de l'Extrême-Orient, de nombreux crustacés et parfois une seule grosse larve de coléoptère les remplissant totalement. Ce même auteur a trouvé des débris organiques et inorganiques et des crustacés dans les utricules d' $U$. bifida, espèce habitant I'Inde, le Japon et la Malaisie, et, dans celles d'U. affinis des mêmes régions, divers animalcules, des diatomées et des nostocacées.

Garbini (1899), sur 610 utricules d'Utricularia neglecta, a trouvé 62 fois la cavité vide, 44 fois des débris indéterminables, 504 fois des organismes variés. Ces derniers, au nombre de 2.084, se répartissent comme il suit : 327 protozoaires, 469 rotifères, 66 nématodes, 1.196 crustacés entomostracés, 13 Gammarus pulex, 16 larves d'insectes, 3 hydrachnes. Les formes les plus fréquentes, au nombre de 1.550 individus, appartiennent seulement à quatre espèces, soit : 195 Stilonychia mytilus Ehrb. (infusoire), 185 Monommata longiseta Bartsch (rotifère), 872 Chydorus sphæricus O. F. Müller (crustacé cladocère) et 298 Cyclops signatus Koch (crustacé copépode).

En 1905, Adolf Eysell, sans donner aucun historique de la question, signale que les plantes d'eau carnivores, et en particulier les utriculaires, peuvent emprisonner des larves et des nymphes de moustiques. Il ajoute que les larves solidement fixées par les itricules, incapables de venir respirer à la surface de l'eau, meurent asphyxiées et qu'un seul pied de cette plante est capable de détruire des centaines de larves. Bien que le travail d'Eysell soit accompagné d'excellentes figures et qu'il ait paru dans le traité classique des maladies tropicales de Mense, il semble avoir échappé à la plupart des auteurs qui, dans leurs travaux les plus récents sur la prophylaxie du paludisme, ne signalent pas le rôle des utriculaires.

En 1923, Carlos França a présenté au Congrès de médecine tropicale de Saint-Paul-de-Loanda un travail original sur ce sujet. Après avoir attiré l'attention sur un petit opuscule de propagande antipaludique publié en russe par Marzinowski, en 1916, dans lequel il est question du rôle des utriculaires, l'auteur nous signale 
la destruction des larves d'Anopheles bifurcatus et de Theobaldia annulata, dans la nature par Utricularia vulgaris, aux environs de Lisbonne (1).

Enfin, en 1925, Victor Apfelbeck, après avoir rappelé que I'Utricularia vulgaris capture de nombreux animaux aquatiques, admet qu'elle peut contribuer à détruire de jeunes larves d'anophèles bien qu'il ait constaté, en Bosnie, un assez grand nombre de ces larves dans des mares renfermant des Utricularia.

Le mécanisme de la capture a été encore peu étudié. En ce qui concerne ce sujet, Mme Treat (1875), de New-Jersey, observant une espèce américaine, l'Utricularia clandestina a été plus heureuse que la plupart des observateurs car elle a pu voir la pénétration de divers animaux (tardigrades, crustacés, larves de moustiques), dans les utricules. Voici en particulier un extrait de son travail cité par Ch. Darwin : "Le Cypris, dit-elle, est très prudent, toutefois il est souvent capturé. Il se place à l'entrée d'une vessie, hésite un instant, puis s'éloigne ; un autre vient tout auprès de la valve, pénètre même dans la dépression, puis se retire comme s'il était effrayé. Un troisième, plus étourdi, ouvre la porte et entre ; mais il n'est pas plutôt à l'intérieur qu'il manifeste quelque inquiétude, il rentre ses pattes et ses antennes et se renferme dans sa coquille. Les larves, probablement celle du cousin (2), qui circulent près de la valve, heurtent la plupart du temps de la tête l'entrée de la prison d'où elles ne peuvent plus sortir. Quelquefois il s'écoule plus de trois ou quatre heures avant qu'une grosse larve ne soit avalée et chaque fois que j'ai assisté à ce spectacle, je n'ai pu m'empêcher de penser à ce qui se passe quand un petit serpent se met en tête d'avaler une grosse grenouille. "

Observations personnelles. - Plusieurs lots d'Utricularia vulgaris (3), récoltés aux environs de Paris, sont placés dans divers réci-

(1) Dans une espèce d'utriculaire des environs de Baltimore le professeur R.-W. Hegner a découvert une euglène verte qui pullule dans les utricules et qu'il a bien voulu me montrer, en octobre 1924, lors de mon voyage d'étude aux Etats-Unis comme hôte de la fondation Rockefeller.

(2) Dans la traduction française de l'ouvrage de Darwin à la page 503 on peut lire: «On a trouvé dans les vessies (de l'Utricularia clandestina) un grand nombre d'animaux capturés: au nombre de ces animaux se trouvaient des crustacés, mais la plupart du temps des larves délicates et allongées. Je suppose que c'étaient des Culicidre ». « Sur quelques tiges, dit Mme Treat, neuf vessies sur dix contenaient ces larves ou leurs restes. Les larves vivaient encore de vingt quatre à trente-six heures après leur emprisonnement, puis elles périssaient n. Il m'est difficile d'affirmer qu'il s'agissait bien de larves de moustiques, mais étant donné l'abondance tout à fait particulière de ces insectes à New-Jersey, avant les magnifiques travaux prophylactiques de la New-Jersey Mosquito extermination association, il est bien probable que c'est Mme Treat qui a constaté la première fois la capture de larves de culicidés par les utriculaires.

(3) Détermination coñfirmée par le $\mathrm{D}^{\mathrm{r}} \mathrm{M}$. Langeron. 
pients renfermant de l'eau de Seine prise au robinet, ou de l'eau de Seine épurée par un séjour de plusieurs mois dans de grandes cuves de verre renfermant diverses plantes aquatiques.

Les utricules des plantes récoltées le 30 avril dans les mares renfermaient quelques débris, mais très peu d'animaux déterminables. En mettant des rameaux jeunes, dont les utricules étaient vides, dans de l'eau renfermant de nombreux crustacés ostracodes, copépodes et cladocères et des hydrachnides, j'ai pu constater, quelques minutes ou quelques heures plus tard, la capture de nombreux petits ostracodes, que leur mode de locomotion à la surface des plantes expose tout particulièrement, et d'un certain nombre de
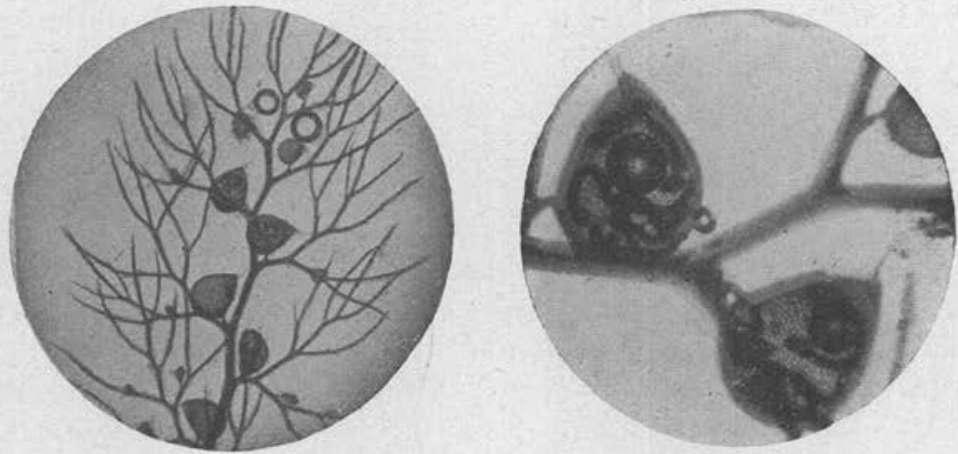

FIG. 1. - A gauche, rameau d'Utricularia vulgaris dont toutes les vésicules renferment de 1 à 5 larves primaires d'Anopheles maculipennis. A droite, deux vésicules vues à un plus fort grossissement montrant chacune 5 larves.

copépodes, parfois des hydrachnides. Je n'ai pas eu l'occasion d'observer l'emprisonnement de daphnies.

En plaçant une centaine de larves d'Anopheles maculipennis, présentant encore leur appareil d'éclosion (1) et mesurant de un à un millimètre et demi de longueur, dans un petit cristallisoir renfermant deux rameaux jeunes d'utriculaire, près d'une cinquantaine furent capturés en moins de trois heures. Dans un exemplaire, on rencontrait en général une seule larve par utricule, mais

(1) L'appareil d'éclosion des larves de moustiques, constitué par une petite dent chitineuse placée sur la face supérieure de la tête de la larve primaire, semble avoir peu retenu l'attention des observateurs. Sauf erreur, c'est F.-W. Edwards qui semble l'avoir signalé le premier en 1919; divers auteurs, tels que Wesenberg-Lund (1920-1921), Dickson Lang (1920) et Séguy l'ont également mentionné. J'ai eu l'occasion de lobserver chez Stegomyia fasciata, divers Culex, Theobaldia annulata et Anopheles maculipennis. Par son grand polymorphisme, cet appareil pourrait être utilisé pour la détermination spécifique des larves primaires des culicidés quand l'étude de ces dernières aura été faite systématiquement. 
dans l'autre exemplaire la plupart des ascidies étaient vides sauf celles d'ın rameau (fig. 1) qui semblait avoir exercé une attraction spéciale sur les larves, dont la majorité des utricules renfermaient de 1 à 5 larves.

J'ai pu répéter les mêmes expériences avec un égal succès en utilisant des larves d'Anopheles maculipennis de 6 à $8 \mathrm{~mm}$. de longueur. Un quart d'heure, parfois une demi-heure après le début de l'expérience, on pouvait voir certaines d'entre elles retenues dans les utricules où, malgré la privation relative d'air (1), elles pouvaient vivre au moins dix heures.

Les larves de Culex apicalis (2) sont également très vite emprisonnées dans les utricules (fig. 2 et 3 ). Dans le courant de la première heure d'expérimentation, près de $50 \mathrm{p}$. cent des larves sont
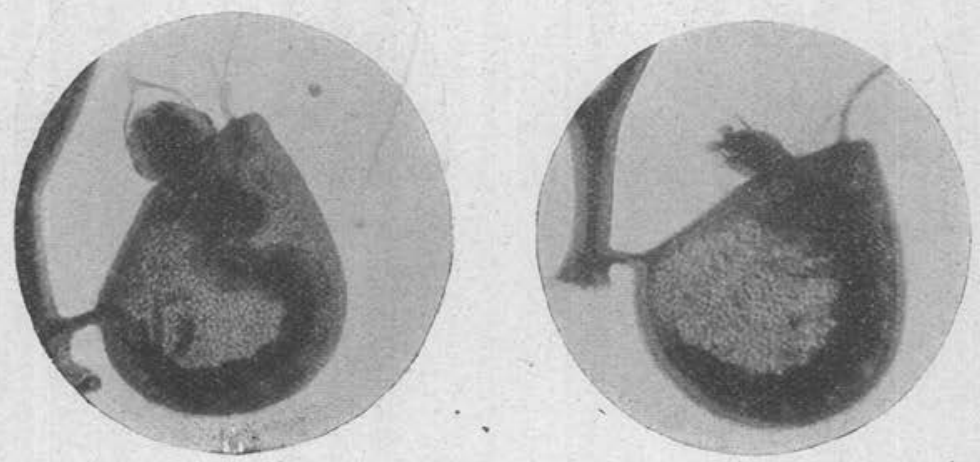

- Fig. 2. - A gauche, une larve adulte de Culex apicalis. A droite, une grosse larve d'Anopheles maculipennis.

capturées. Le temps m'a manqué pour étudier plus complètement ce fait et pour établir si les larves non capturées présentent des tropismes particuliers qui les éloignent des utriculaires ou si, tout simplement, ce sont les utricules attractives, saturées de larves, qui ne peuvent plus en absorber.

J'aurais voulu élucider par quel mécanisme les larves de culicidés entrent dans les utricules car toutes celles que j'ai eu l'occasion d'étudier, étaient prises par la partie postérieure du corps (lamelles branchiales, derniers anneaux du corps), ou par le corps

(1) Les utricules renferment en effet très souvent une grosse bulle de gaz, signalée dès 1848 par Benjamin, facile à voir sur les microgrophotographies jointes à ce travail. Ce gaz doit être de l'oxygène car il se forme parfois rapidement sous l'influence de la lumière solaire.

(2) Détermination du Dr H. Galliard, préparateur au Laboratoire de Parasitologie. 
entier, la tête restant au dehors (fig. 2 et 3 ). Les photographies publiées par A. Eysell, dès 1905, montrent aussi les aspects que je viens de signaler ci-dessus.

Si réellement les larves entrent dans l'utricule par leur extrémité postérieure, on peut se demander si elles ne sont pas attirées par la présence fréquente de cette bulle d'oxygène signalée ci-dessus. Ellles obćiraient ainsi à un tropisme comparable à celui des larves de Mansonia, de Mansonioides et de Tæniorhynchus qui enfoncent leur siphon respiratoire dans les tissus des plantes aquatiques pour en absorber l'oxygène, ou comparable à celui des curieuses larves d'un Uranotænia (1), que je viens d'observer à Manaos (Amazonie), larves qui ne présentenit aucune adaptation particulière du siphon comme dans les genres signalés ci-dessus. Ces larves ne viennent
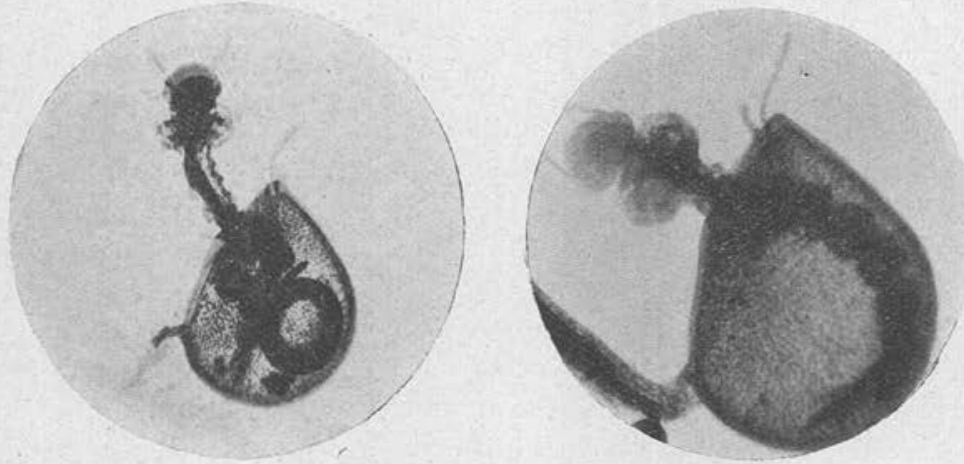

FIG. 3. - A gauche et à droite, larves adultes de Culex apicalis capturées par Utricularia vulgaris. La vésicule de gauche renferme une grosse bulle de gaz.

jamais respirer l'air à la surface de l'eau, elles le prennent entre les poils des feuilles de diverses plantes aquatiques quand ceux-ci en retiennent comme dans les cas de Pistia stratioides, ou encore au moment où diverses plantes aquatiques émettent de l'oxygène au niveau de leurs parties immergées.

Quel que soit le mécanisme qui explique la capture des larves de culicidés, un fait bien établi c'est que les utriculaires capturent facilement ces animaux et possèdent dans certains cas une capacité destructrice considérable. Comme, d'autre part, les larves de l'Anopheles maculipennis, principal vecteur du paludisme en Europe et dans le bassin méditerranéen, apparaissent après les utriculaires et disparaissent, soit simultanément, soit après, ces

(1) Détermination du Dr F. Larrousse, préparateur au Laboratoire de Parasitologie. 
plantes peuvent détruire un nombre de larves qui n'est certainement pas négligeable (1). Il y a un grand intérêt à étudier les conditions physico-chimiques qui déterminent le développement des utriculaires et à tenter leur acclimatation dans les eaux douces des régions à paludisme où elles n'existent pas encore.

L'utilisation des animaux et des végétaux auxiliaires peut rendre de grands services dans la lutte contre les animaux nuisibles. Cependant, avant d'introduire dans un pays donné de nouveaux êtres vivants, il est indispensable que des biologistes compétents étudient au préalable les répercussions qui pourraient éventuellevent se produire sur la faune et la flore autochtone. Dans le cas des utriculaires aucune intervention fâcheuse ne paraît à craindre.

\section{RÉSUMÉ}

1. La capture des larves de culicidés par les vésicules des utriculaires, signalée d'abord par Mme Treat de New-Jersey, en 1875, revue par Eysell en 1905 et par França en 1923, a été constatée expérimentalement par moi dans le cas d'Anopheles maculipennis et de Culex apicalis.

2. Comme les utriculaires présentent parfois une grande efficacité destructrice, il y aurait intérêt à bien connaître les conditions qui favorisent leur acclimatation et leur pullulation, afin de s'en servir dans la prophylaxie du paludisme où tous les moyens, surtout ceux d'ordre biologique, toujours les plus économiques, doivent être utilisés.

\section{Bibliographie}

APFELBEck (V.). - Recherches et observations sur les Arthropodes pathogènes de l'homme el des animaux. Edition de l'Inspectorat du Ministère de la Santé publique. Sarajevo, No 17, 1925.

Benjamin. - Ueber den Bau and die Physiologie der Utricularieæ. Bot. Zeit., VI, 1848.

Coнn. - Ueber die Function der Blasen von Aldrovanda und Utricularia Beiträge zur Biologie der Pflanzen, I, 3, 1875.

Crouan (P.-E. et H.-M.). - Florule du Finistère, Brest, 1867.

(1) Je dois signaler cependant qu'à Porto-Vecchio (Corse), je n'ai rencontré ni larve d'Anopheles ni larve de Culex dans les vésicules de divers pieds d'Utricularia vulgaris provenant d'une mare où les culicidés se rencontrait d'ailleurs en petit nombre, le 6 juillet 1925 . 
Darwin (Ch.). - Insectivorous Plants. 1875. Traduction Barbier. Reinwald et Cie, Paris, 1877.

Delpino. - Ult. Osservaz. Sulla Dicogamia, 1868-1869, p. 16 (cité par Darwin).

Eysell (A.). - Die Stechmücken. Handbuch der Tropenkrankeiten de Mense, II, p. 80 , pl. III, 1905.

França (C.). - L'emploi des plantes dans Ie combat des moustiqués. Congrès de médecine tropicale de Saint-Paul de Loanda. Revista medica de Angola, III, avril 1923, p. 419.

Garbini. - Le vittime della Utricularia neglecta. Venezia, C. Ferrari, 1899.

Goeber (K.). - Morphologische und biologische Studien. Ann. du Jardin botanique de Builenzorg, IX, 1890.

Holland, - Quart. Mag. of the Nat. Hist. Soc. High Wycombe, juillet 1868, p. 5 (cité par Darwin).

Manzinowski (E.). - La lutte contre la malaria (en russe). Opuscule de 19 pages, 3 pl., Moscou, 1916.

Schimper. - Notizen ueber Insectenfressende Pflanzen. Bot. Zeit., XV, 1882, p. 241-248, pl. IV.

Treat. - New-York Tribune. Reproduit dans Gardener's Chron., 1875, p. 303.

Laboratoire de Parasitologie de la Facnlté de médeclne de Paris. 